\title{
Amplitude control approach for chaotic signals
}

\author{
Chunbiao Li · J.C. Sprott
}

Received: 15 November 2012 / Accepted: 13 March 2013 / Published online: 23 March 2013

(C) The Author(s) 2013. This article is published with open access at Springerlink.com

\begin{abstract}
A general approach based on the introduction of a control function for constructing amplitudecontrollable chaotic systems with quadratic nonlinearities is discussed in this paper. We consider three control regimes where the control functions are applied to different coefficients of the quadratic terms in a dynamical system. The approach is illustrated using the Lorenz system as a typical example. It is proved that wherever control functions are introduced, the amplitude of the chaotic signals can be controlled without altering the Lyapunov exponent spectrum.
\end{abstract}

Keywords Amplitude control - Lyapunov exponent spectrum $\cdot$ Lorenz system

C. Li $(\varangle)$

Engineering Technology Research and Development

Center of Jiangsu Circulation Modernization Sensor

Network, Jiangsu Institute of Economics and Trade

Technology, 104 Shimenkan St., Guanghua Rd.,

Nanjing 210007, China

e-mail: chunbiaolee@gmail.com

C. Li · J.C. Sprott

Department of Physics, University of Wisconsin-Madison, Madison, WI 53706, USA

C. $\mathrm{Li}$

School of Information Science and Engineering, Southeast University, Nanjing 210096, China

\section{Introduction}

Chaos is a well-known phenomenon in physics, engineering, and many other scientific disciplines. Recently, simple chaotic flows and circuits have aroused much interest because of their potential applications [1-5]. Meanwhile, the amplitude control of a chaotic signal is important in applications of chaos. Furthermore, it turns out that the amplitude control technique described here not only yields the expected amplification without any extra circuitry but also provides a new security encoding key.

Perhaps the simplest example of amplitude control occurs in piecewise-linear systems with a single constant term, in which case the value of the constant controls the amplitude of all the variables without altering the Lyapunov exponent spectrum. Li et al. [6-9] have proposed several such chaotic systems using the absolute value nonlinearity. Another common piecewiselinear function is the signum, which switches from -1 to +1 as its argument goes from negative to positive. If it is the only nonlinearity and there are no constant terms, then its coefficient can provide amplitude control since it uniquely determines the scale of the variables. If the signum is multiplied by one of the variables so that the signum determines only the sign of the corresponding term and not its magnitude, then a constant term is also needed to provide amplitude control.

In this paper we discuss a general approach to controlling the amplitude in chaotic systems with 
quadratic nonlinearities, which are commonly studied, but for which amplitude control is more problematic. Unlike most of the schemes proposed in the literature, we consider cases where the variables are simultaneously controlled in proportion to one another, rather than the more usual cases in which they are independently controlled, which generally requires additional circuitry $[10,11]$. This result is accomplished through the judicious choice of the coefficients of a quadratic term to achieve amplitude control. The amplitude control approach works for any dynamical behavior including limit cycles and chaos. In Sect. 2, we discuss the amplitude control mechanism. In Sect. 3, we apply the amplitude control technique to the Lorenz system, showing the details of three control regimes. The general amplitude control approach is discussed in Sect. 4.

\section{Amplitude control mechanism}

A chaotic system with quadratic nonlinearities and no constant terms can be written as

$\dot{\mathbf{X}}=\sum_{\|\mathbf{p}\|_{1}=1} g_{\mathbf{p}}(\mathbf{X})+\sum_{\|\mathbf{p}\|_{1}=2} g_{\mathbf{p}}(\mathbf{X})$

where $\mathbf{X}$ is a state variable vector, $\mathbf{X}=\left(x_{1}, x_{2}, \ldots, x_{l}\right)^{T}$, $\mathbf{p}$ is an index vector of the state variable, and $\mathbf{p}=$ $\left(p_{1}, p_{2}, \ldots, p_{l}\right), p_{i} \geqq 0(i=1,2, \ldots, l)$, where $p_{i}$ are integers. $\|\mathbf{p}\|_{1}$ means vector 1 norm. $g_{\mathbf{p}}(\mathbf{X})=$ $c_{[\mathbf{p}]}^{k} x_{1}^{p_{1}} x_{2}^{p_{2}} \cdots x_{l}^{p_{l}}, c_{[\mathbf{p}]}^{k}(k=1,2, \ldots, l)$ is the coefficient of each quadratic term in the dimension $k$. Therefore, the introduced function $F=b_{k} f(m)$ in the quadratic terms in Eq. (2) can control the amplitude of the signal variable vector $\mathbf{X}$,

$\dot{\mathbf{X}}=\sum_{\|\mathbf{p}\|_{1}=1} g_{\mathbf{p}}(\mathbf{X})+\sum_{\|\mathbf{p}\|_{1}=2} F g_{\mathbf{p}}(\mathbf{X})$,

where $m$ is an ultimate physical input and $b_{k}=0$ or 1 $(k=1,2, \ldots, l)$. The fundamental law for amplitude control is that a simultaneous change in the coefficients of all the nonlinear terms leads to a system of equations that can be reduced to the original equations by a suitable linear rescaling of the variables.

(1) Total Amplitude Control (TAC). If the unified control functions are introduced into the coefficients of all the quadratic terms, the amplitude of all the variables will be controlled. This is called TAC.

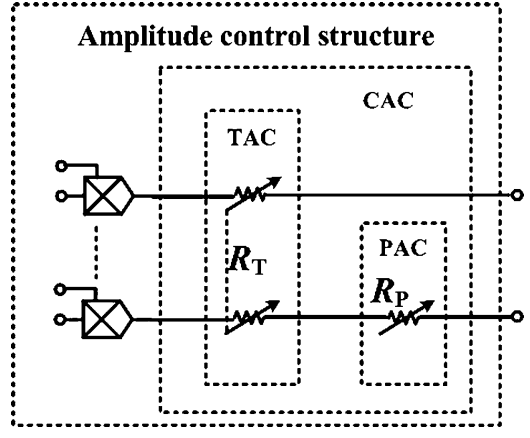

Fig. 1 Amplitude control structure

(2) Partial Amplitude Control (PAC). When the unified control functions are introduced into only some of the quadratic terms, then only some of the variables can be controlled, while the amplitude of the other variables remains unchanged. Not every chaotic system allows PAC since it depends on the location of the linear terms in the system equations. In the PAC mode, it may be possible to control more than one variable simultaneously with a single control function.

(3) Composite Amplitude Control (CAC). If the amplitude control is a mixture of TAC and PAC, we call this mode CAC. In this condition, the introduced control functions are different for the different quadratic terms. The result is that the amplitudes of the variables are individually controlled.

In practice, the introduction of a control function only requires a potentiometer to replace the fixed resistor in an electrical circuit implementation. Ganged potentiometers can be used to obtain the simultaneous control of multiple parameters. Figure 1 shows the amplitude control structure based on a control function in the quadratic coefficients. As illustrated in the diagram, the substitution of a variable resistor can make the signals generated in a chaotic circuit adjustable.

To illustrate this control mechanism, we apply different control modes to the well-known Lorenz system [12-14] by introducing control functions in the quadratic nonlinearities. From the analysis of the Lorenz system in different control regimes, it is proved that such an amplitude control function does not affect the Lyapunov exponent spectrum. 


\section{Amplitude control regime}

\subsection{Partial Amplitude Control (PAC)}

The Lorenz system [12] is the most famous chaotic system with quadratic nonlinearities and provides a good and non-trivial example for control since it includes two quadratic terms given by

$\left\{\begin{array}{l}\dot{x}=a(y-x), \\ \dot{y}=-x z+r x-y, \\ \dot{z}=x y-b z,\end{array}\right.$

where typically $a=10, b=8 / 3$, and $r=28$ for chaos. We can introduce the control function in the coefficient of the quadratic term in either the second or third dimension. In fact, only when the control function is introduced into the third dimension can one achieve PAC. Assuming that the variable $z$ is unchanged, through a simple linear transformation of the other variables, the system with a new coefficient of the $x y$ term can be transformed back to the original equations. However, if the control function is introduced in the $x z$ term of the second dimension, no linear transformation of the variables can restore the original equations. The reason the former works is that in the first two equations, each term depends on the first power of the variables $x$ and $y$. Furthermore, this linear dependence of the first two equations means that PAC cannot be obtained by controlling any of the linear terms without introducing additional parameters.

Theorem 1 An introduced control function $f(m)$ in the quadratic term in the third dimension of the Lorenz system can realize PAC. The controlled equation is

$\left\{\begin{array}{l}\dot{x}=a(y-x), \\ \dot{y}=-x z+r x-y, \\ \dot{z}=f(m) x y-b z .\end{array}\right.$

The function $f(m)$ controls the amplitude of the variables $x$ and $y$ according to $1 / \sqrt{f(m)}(f(m)>0)$ while the amplitude of variable $z$ is unchanged, and the Lyapunov exponent spectrum remains unchanged when the control function varies.

Proof Let $u=x / \sqrt{f(m)}, v=y / \sqrt{f(m)}, w=z$. The resulting system,

$$
\left\{\begin{array}{l}
\dot{u}=a(v-u) \\
\dot{v}=-u w+r u-v, \\
\dot{w}=u v-b w
\end{array}\right.
$$

is identical to (3). Therefore, the function $f(m)$ can control the amplitude of the variables $x$ and $y$ according to $1 / \sqrt{f(m)}$, while the amplitude of $z$ is unchanged.

For system (4), the rate of state space volume contraction is given by $\nabla V=\frac{\partial \dot{x}}{\partial x}+\frac{\partial \dot{y}}{\partial y}+\frac{\partial \dot{z}}{\partial z}=-a-$ $b-1$ and is unchanged by the function $f(m)$. System (4) possesses three equilibrium points. Let $\alpha=$ $\sqrt{b(r-1)}, \beta=\sqrt{f(m)}, \omega=r-1$. Then there are three equilibria at $P_{1}=(0,0,0)=(0 / \beta, 0 / \beta, 0)$, $P_{2}=(\alpha / \beta, \alpha / \beta, \omega)$, and $P_{3}=(-\alpha / \beta,-\alpha / \beta, \omega)$. The control function moves the equilibrium points along the $x$-axis and $y$-axis in a similar way without changing the $z$-axis coordinate.

The Jacobian matrix of system (4) is given by

$J=\left(\begin{array}{ccc}-a & a & 0 \\ r-z & -1 & -x \\ f(m) y & f(m) x & -b\end{array}\right)$,

whose characteristic equation is

$$
\begin{aligned}
\lambda^{3} & +(a+b+1) \lambda^{2}+(a+b+a b-a r+a z \\
& \left.+f(m) x^{2}\right) \lambda+a b-a b r+a b z+a f(m) x^{2} \\
& +a f(m) x y=0 .
\end{aligned}
$$

This equation shows the effect of the control function on the eigenvalues. When the coordinate shift in the $x$-axis and $y$-axis is $u=x / \sqrt{f(m)}, v=y / \sqrt{f(m)}$, $w=z$, the characteristic roots remain the same as before, proving that the Lyapunov exponent spectrum is unchanged.

In practical engineering, the control function is realized by a potentiometer. The relationship between resistance and the rotation angle $m$ will lead to different control functions. If $f(m)=m$, the amplitude of variables $x$ and $y$ will vary according to $1 / \sqrt{m}$ while variable $z$ is unchanged as shown in Fig. 2. If $f(m)=$ $e^{m}$, the amplitude of variables $x$ and $y$ will vary according to $1 / \sqrt{e^{m}}$ as shown in Fig. 3. Figures 2(a), 3 (a) give the maxima and minima of $x, y$, and $z$, which shows that the amplitude $x$ and $y$ change according to $1 / \sqrt{m}$ and $1 / \sqrt{e^{m}}$, respectively, while the amplitude of $z$ remains unchanged. Figures 2(b), 3(b) show the constant Lyapunov exponents as $m$ changes to within numerical error. Because the exponent function is positive, the range of the control parameter is selected as $[-2,2]$. 
Fig. 2 The amplitude control diagram in system (4) in the region $m \in[0.1,20.1]$ with initial conditions

$\left(x_{0} ; y_{0} ; z_{0}\right)=(0 ; 1 ; 0)$ when $f(m)=m$ : (a) signal amplitude, (b) Lyapunov exponents

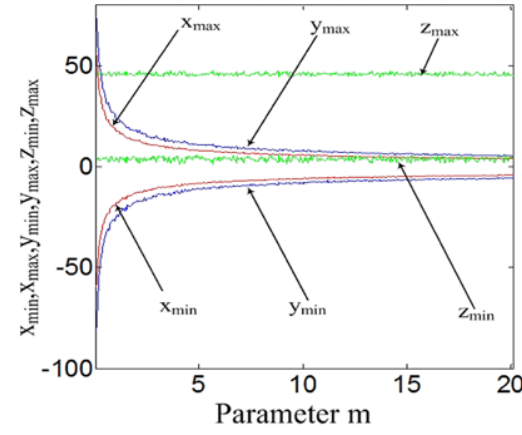

(a)

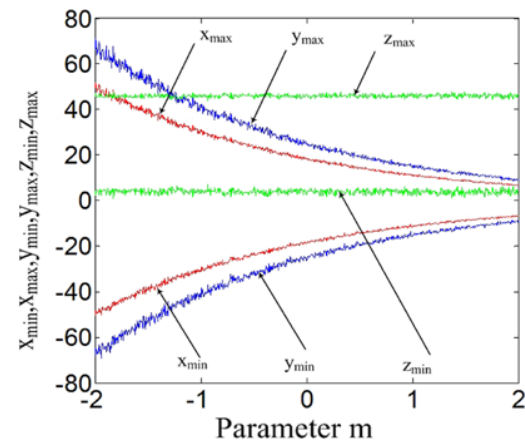

(a)

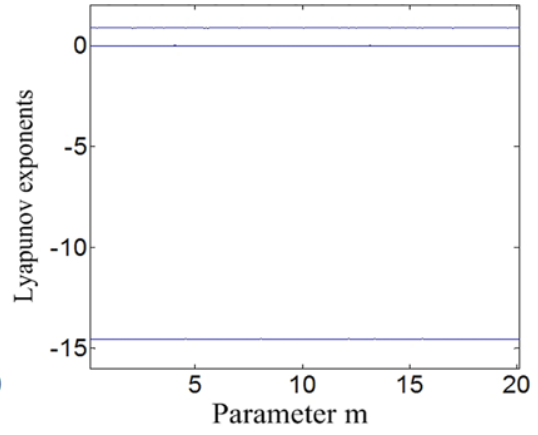

(b)

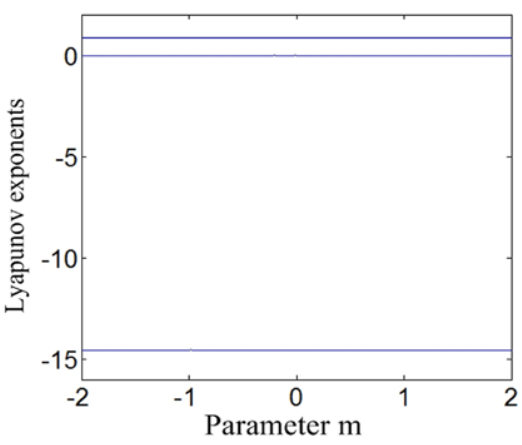

(b)

\subsection{Total Amplitude Control (TAC)}

Theorem 2 The same two functions applied to both quadratic terms,

$\left\{\begin{array}{l}\dot{x}=a(y-x), \\ \dot{y}=-f(m) x z+r x-y, \\ \dot{z}=f(m) x y-b z,\end{array}\right.$

can control the amplitude of all three variables $x, y$, and $z$ according to $1 / f(m)$ while the Lyapunov exponent spectrum remains unchanged.

Proof Let $u=x / f(m), v=y / f(m), w=z / f(m)$. Then the resulting equations in the variables $u, v, w$ are identical to Eq. (3). Therefore, the function $f(m)$ controls the amplitude of all variables according to $1 / f(m)$. The function need not be positive in this case. It can be negative, but it cannot be zero, else the variables will be unbounded.

For system (8), we still have $\nabla V=\frac{\partial \dot{x}}{\partial x}+\frac{\partial \dot{y}}{\partial y}+\frac{\partial \dot{z}}{\partial z}=$ $-a-b-1$ independent of $f(m)$. System (8) has three equilibrium points. For $\alpha=\sqrt{b(r-1)}, \delta=f(m)$, $\omega=r-1$, the equilibria are at $P_{1}=(0,0,0)=$
$(0 / \delta, 0 / \delta, 0 / \delta), \quad P_{2}=(\alpha / \delta, \alpha / \delta, \omega / \delta), \quad P_{3}=$ $(-\alpha / \delta,-\alpha / \delta, \omega / \delta)$. The control function moves the equilibrium points along the $x$-axis, $y$-axis and $z$-axis proportionally.

The Jacobian matrix of system (8) is given by

$J=\left(\begin{array}{ccc}-a & a & 0 \\ r-f(m) z & -1 & -f(m) x \\ f(m) y & f(m) x & -b\end{array}\right)$,

whose characteristic equation is

$$
\begin{aligned}
\lambda^{3} & +(a+b+1) \lambda^{2}+(a+b+a b-a r+a f(m) z \\
& \left.+f^{2}(m) x^{2}\right) \lambda+a b-a b r+a b f(m) z \\
& +a f^{2}(m) x^{2}+a f^{2}(m) x y=0 .
\end{aligned}
$$

The control function in the characteristic equation is canceled by the coordinate shift in the $x$-axis, $y$-axis and $z$-axis. With the coordinate transformation $u=x / f(m), v=y / f(m), w=z / f(m)$, the characteristic roots remain unchanged as before. Therefore, the Lyapunov exponent spectrum remains unchanged.

Suppose the control function is $f(m)=m$. Then from Theorem 2, the amplitude of variables $x, y$, and 
Fig. 4 The amplitude control diagram in system (8) in the region $m \in[-4,6]$ with initial conditions

$\left(x_{0} ; y_{0} ; z_{0}\right)=(0 ; 1 ; 0)$ when $f(m)=m$ : (a) signal amplitude, (b) Lyapunov exponents

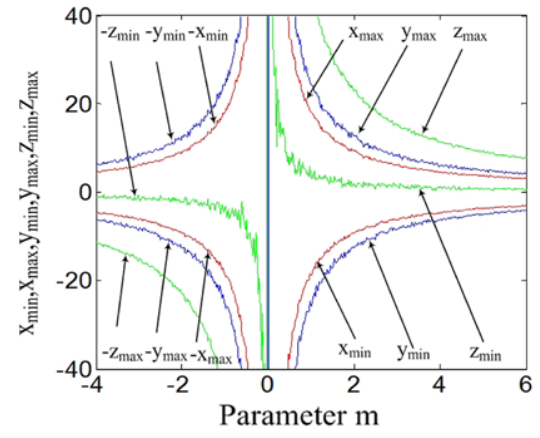

(a)

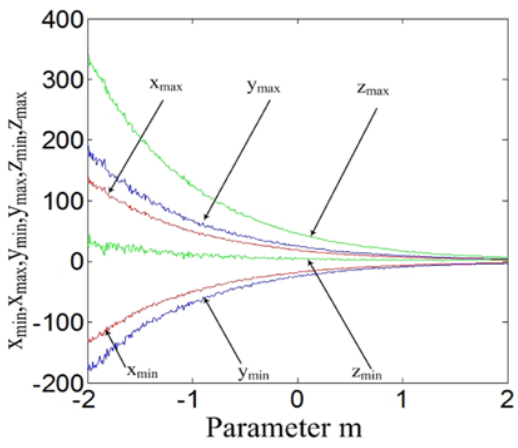

(a)

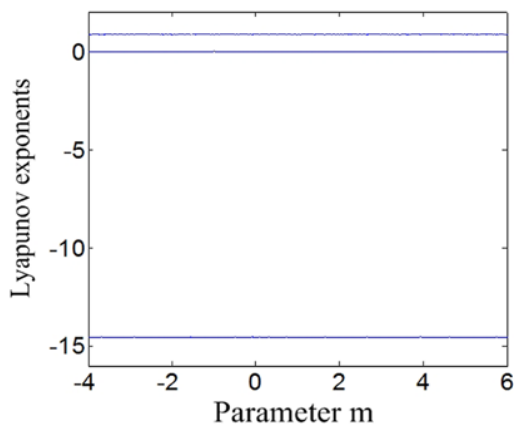

(b)

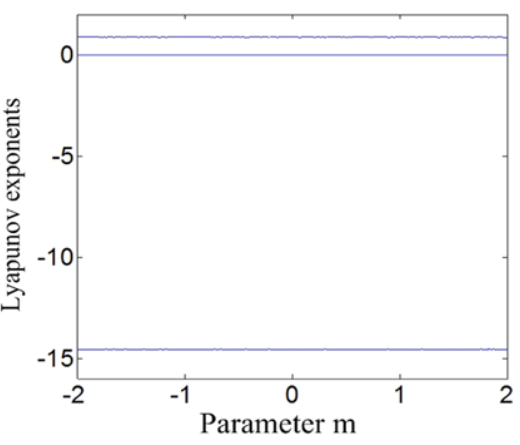

(b) $z$ will vary according to $1 / \mathrm{m}$. To show that the control function can be negative, let it vary from -4 to 6 . If $f(m)=e^{m}$, the amplitude of variables $x, y$, and $z$ will vary according to $1 / e^{m}$. The region of parameter $m$ is selected as $[-2,2]$. Figures 4(a), 5(a) give the maxima and minima of the variables $x, y$, and $z$ which shows that the amplitudes of all variables are controlled to change according to $1 / m$ or $1 / e^{m}$, respectively. Figures 4(b), 5(b) indicate that $f(m)$ does not change the Lyapunov exponents.

\subsection{Composite Amplitude Control (CAC)}

Theorem 3 The two different control functions $f(m)$ and $g(n)$ are introduced into the coefficients of the quadratic terms in system (3) as follows:

$\left\{\begin{array}{l}\dot{x}=a(y-x), \\ \dot{y}=-f(m) x z+r x-y, \\ \dot{z}=(f(m)+g(n)) x y-b z,\end{array}\right.$

These functions can control the amplitude of variables $x$ and $y$ according to $\frac{1}{\sqrt{f(m)(f(m)+g(n))}}$ and $z$ accord- ing to $\frac{1}{f(m)}$, while the Lyapunov exponent spectrum remains unchanged.

Proof By the variable transformations,

$$
\left\{\begin{array}{l}
u=\frac{x}{\sqrt{f(m)(f(m)+g(n))}}, \\
v=\frac{y}{\sqrt{f(m)(f(m)+g(n))}} \\
w=\frac{z}{f(m)} .
\end{array}\right.
$$

Equation (11) will be the same as Eq. (3). Thus the control functions $f(m)$ and $g(n)$ can control the amplitude of variables $x$ and $y$ according to $\frac{1}{\sqrt{f(m)(f(m)+g(n))}}$, and $z$ according to $\frac{1}{f(m)}$.

For system (11), the state space contraction is $\nabla V=\frac{\partial \dot{x}}{\partial x}+\frac{\partial \dot{y}}{\partial y}+\frac{\partial \dot{z}}{\partial z}=-a-b-1$, independent of $f(m)$ and $g(n)$. System (11) has three equilibrium points. For $\alpha=\sqrt{b(r-1)}, \beta=\sqrt{f(m)}$, $\delta=f(m), \varphi=\sqrt{f(m)+g(n)}, \omega=r-1$, the equilibria are at $P_{1}=(0,0,0)=(0 / \beta \varphi, 0 / \beta \varphi, 0 / \delta), P_{2}=$ $(\alpha / \beta \varphi, \alpha / \beta \varphi, \omega / \delta), \quad P_{3}=(-\alpha / \beta \varphi,-\alpha / \beta \varphi, \omega / \delta)$. The control function moves the equilibrium points 
Fig. 6 The amplitude control diagram in system (11) in the region $m \in[0.1$, $10.1]$ with initial conditions $\left(x_{0} ; y_{0} ; z_{0}\right)=(0 ; 1 ; 0)$ when $f(m)=1 / m$, $g(m)=e^{m}:$ (a) signal amplitude, (b) Lyapunov exponents

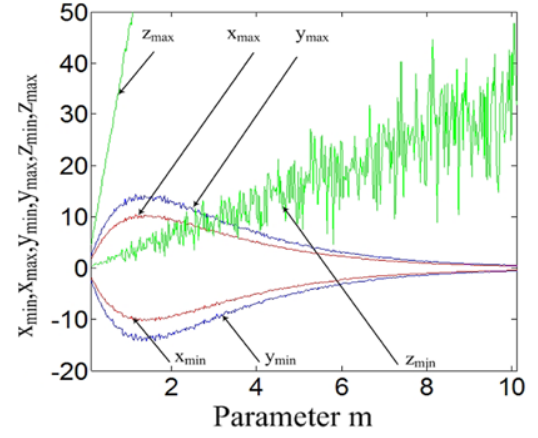

(a)

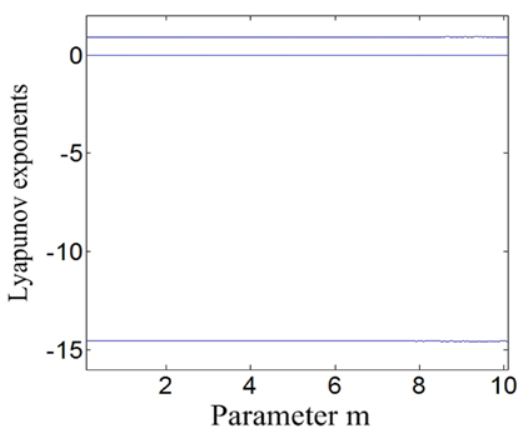

(b) along the $x$-axis, $y$-axis, and $z$-axis according to $1 / \beta \varphi$, $1 / \beta \varphi, 1 / \delta$, respectively.

The Jacobian matrix of system (11), is given by

$J=\left(\begin{array}{ccc}-a & a & 0 \\ r-f(m) z & -1 & -f(m) x \\ (f(m)+g(n)) y & (f(m)+g(n)) x & -b\end{array}\right)$.

Therefore, the characteristic equation is

$$
\begin{aligned}
\lambda^{3} & +(a+b+1) \lambda^{2}+(a+b+a b-a r+a f(m) z \\
& \left.+f(m)(f(m)+g(n)) x^{2}\right) \lambda+a b-a b r \\
& +a b f(m) z+a f(m)(f(m)+g(n)) x^{2} \\
& +a f(m)(f(m)+g(n)) x y=0 .
\end{aligned}
$$

The effect of the control function in the characteristic equation is canceled by the coordinate shift in the $x$-axis, $y$-axis, and $z$-axis. When a coordinate transformation of the chaotic flow is executed as in Eq. (12), the characteristic roots remain constant as before. These factors lead to a constant Lyapunov exponent spectrum.

Suppose the control function $f(m)$ and the control function $g(n)$ share the same ganged potentiometers with a common physical input $(m=n)$. Let $f(m)=$ $1 / m, g(m)=e^{m}$. Then according to Theorem 3, the amplitude of variables $x$ and $y$ will vary according to $\frac{m}{\sqrt{1+m e^{m}}}$, while $z$ will vary according to $m$.

Figure 6(a) shows the maxima and minima of the variables $x, y$, and $z$, confirming that the amplitude of variables $x$ and $y$ are controlled according to $\frac{m}{\sqrt{1+m e^{m}}}$, while $z$ is controlled according to $m$. Figure $6(\mathrm{~b})$ confirms that the control functions $f(m)$ and $g(m)$ do not influence the Lyapunov exponents.

\section{Conclusions and discussion}

In conclusion, we have presented a general approach by introducing control functions into the quadratic terms of a dynamical system to realize amplitude control of chaotic signals and discussed its three realization regimes in which the amplitude can be partially or totally controlled. In the composite mode and partial mode, there are different amplitude variations for the variables. These control approaches can be realized in an electrical circuit by a single potentiometer or by ganged potentiometers, which are convenient and benefit secure communications and other fields of information engineering.

Acknowledgements C. Li wishes to thank Z. Zhou for mathematical assistance. The research described in this publication was made possible in part by Grant Nos. 2011M500838, 2012 T50456 and 1002004C from the Science Foundation for Postdoctoral Program of People's Republic of China. In addition, this work was partially supported by the Jiangsu Overseas Research \& Training Program for University Prominent Young and Middle-aged Teachers and Presidents, the 4th 333 Highlevel Personnel Training Project (Su Talent [2011] No. 15) and Qing Lan Project of Jiangsu Province.

Open Access This article is distributed under the terms of the Creative Commons Attribution License which permits any use, distribution, and reproduction in any medium, provided the original author(s) and the source are credited.

\section{References}

1. Sprott, J.C.: Some simple chaotic flows. Phys. Rev. E, Stat. Nonlinear Soft Matter Phys. 50, R647-R650 (1994)

2. Sprott, J.C.: Some simple chaotic jerk functions. Am. J. Phys. 65, 537-543 (1997)

3. Sprott, J.C.: Simple chaotic systems and circuits. Am. J. Phys. 68, 758-763 (2000)

4. Sprott, J.C.: Simple models of complex chaotic systems. Am. J. Phys. 76, 474-480 (2008) 
5. Piper, J.R., Sprott, J.C.: Simple autonomous chaotic circuits. IEEE Trans. Circuits Syst. II, Analog Digit. Signal Process. 57, 730-734 (2010)

6. Li, C.B., Wang, D.: An attractor with invariable Lyapunov exponent spectrum and its Jerk circuit implementation. Acta Phys. Sin. 58, 764-770 (2009)

7. Li, C.B., Wang, H.-K., Chen, S.: A novel chaotic attractor with constant Lyapunov exponent spectrum and its circuit implementation. Acta Phys. Sin. 59, 783-791 (2010)

8. Li, C.B., Wang, J., Hu, W.: Absolute term introduced to rebuild the chaotic attractor with constant Lyapunov exponent spectrum. Nonlinear Dyn. 68, 575-587 (2012)

9. Li, C.B., Xu, K.S., Hu, W.: Sprott system locked on chaos with constant Lyapunov exponent spectrum and its antisynchronization. Acta Phys. Sin. 60(12), 120504 (2011)
10. Cuomo, K.M., Oppenheim, A.V.: Circuit implementation of synchronized chaos with applications to communications. Phys. Rev. Lett. 71(1), 65-68 (1993)

11. Zhang, X., Chen, J., Peng, J.: Theoretical and experimental study of Chen chaotic system with notch filter feedback control. Chin. Phys. B 19(9), 090507 (2010)

12. Lorenz, E.N.: Deterministic nonperiodic flow. J. Atmos. Sci. 20, 130-141 (1963)

13. Curry, J.H.: A generalized Lorenz system. Commun. Math. Phys. 60(3), 193-204 (1978)

14. Lü, J., Chen, G., Cheng, D., Celikovsky, S.: Bridge the gap between the Lorenz system and the Chen system. Int. J. Bifurc. Chaos Appl. Sci. Eng. 12, 2917 (2002) 